\title{
Application of Deep Learning Image Reconstruction (DLIR) Algorithm to Improve Image Quality in CT Angiography of Children with Takayasu Arteritis
}

Jihang Sun

Beijing Children's Hospital

Haoyan Li

Beijing Children's Hospital

Jianying Li

General Electric (United States)

Haiyun Li

Capital Medical University

Michelle Li

Stanford University

Zuofu Zhou ( 464481492@qq.com )

Fujian Provincial Maternity and Children's Hospital

\section{Yun Peng}

Beijing Children's Hospital

\section{Research Article}

Keywords: Computed Tomography Angiography, Takayasu Arteritis, Pediatrics, Deep Learning

Posted Date: September 21st, 2021

DOl: https://doi.org/10.21203/rs.3.rs-805973/v1

License: (9) This work is licensed under a Creative Commons Attribution 4.0 International License.

Read Full License

Version of Record: A version of this preprint was published at Journal of X-Ray Science and Technology on November 18th, 2021. See the published version at https://doi.org/10.3233/XST-211033. 


\section{Abstract}

purpose: To evaluate the image quality improvement in CTA of children with Takayasu arteritis (TAK) using a Deep learning image reconstruction (DLIR) in comparison to other reconstruction algorithms.

Methods: 32 patients $(9.14 \pm 4.51$ years old) with TAK underwent neck, chest and abdominal CTA with $100 \mathrm{kVp}$ were enrolled. Images were reconstructed at $0.625 \mathrm{~mm}$ slice thickness using Filtered BackProjection (FBP), 50\% adaptive statistical iterative reconstruction-V (ASIR-V), 100\%ASIR-V and DLIR with high setting (DLIR-H). The CT number and standard deviation (SD) of the descending aorta and back muscle were measured and contrast-to-noise ratio (CNR) for aorta was calculated. The vessel visualization, overall image noise and diagnostic confidence were evaluated using a 5-point scale (5, excellent; 3 , acceptable) by 2 observers.

Results: There was no significant difference in CT number across all reconstructions. The image noise values (in HU) were $31.36 \pm 6.01,24.96 \pm 4.69,18.46 \pm 3.91$ and $15.58 \pm 3.65$, and CNR values for aorta were $11.93 \pm 2.12,15.66 \pm 2.37,22.54 \pm 3.34$ and $24.02 \pm 4.55$ with FBP, 50\%ASIR-V, $100 \% A S I R-V$ and DLIR-H, respectively. The $100 \% A S I R-V$ and DLIR-H images had similar noise and CNR (all P>0.05), and both had lower noise and higher CNR than FBP and 50\%ASIR-V images (all $P<0.05$ ). The subjective evaluation suggested that all images were diagnostic for large arteries, but only 50\%ASIR-V and DLIR-H met the diagnostic requirement for small arteries $(3.03 \pm 0.18$ and $3.53 \pm 0.51)$.

Conclusions: DLIR-H improves the CTA image quality and diagnostic confidence for TAK patients compared with $50 \% A S I R-V$, and best balances image noise and spatial resolution compared with $100 \% A S I R-V$.

\section{Background}

CT angiography (CTA) is a common examination, which can quickly and noninvasively identify the main artery malformation (1-7) and has been widely used for children. It is also an important evaluation method for Takayasu arteritis (TAK) (8-9). Children with TAK need to use immunosuppressants, the inflammatory indexes of children with TAK usually tend to be normal immediately after treatment, which cannot correctly reflect the severity and activity of the disease $(8,10)$. Therefore, CTA has become an important method to evaluate the status of TAK and sometime is even more sensitive than laboratory test results, especially for patients after using immunosuppressants (8-11). However, children are radiationsensitive and CT scans should be performed following the as low as reasonably achievable (ALARA) principle (12). How to maintain or even improve the image quality when the radiation dose decreases is worthy of continuous study. Iterative reconstruction (IR) algorithms have shown the ability to reduce image noise and have contributed to the radiation dose reduction in CTA for the last decade (13-15). However, constrained by the modelling complexity, there is a need to balance spatial resolution and image noise in IR algorithms, and IR with high weights are often limited in clinical applications to avoid blurring artifacts or blotchy appearance in IR images, which affects the efficiency of dose reduction (16- 
17). Recently, a deep learning image reconstruction (DLIR) algorithm (GE Healthcare) has been developed based on the artificial intelligence and deep neural networks. The current version of DLIR consists of three different strength settings: low, medium, and high to yield different noise reduction capability. Studies have shown that DLIR at different strengths can further reduce image noise while avoiding the blurring artifacts, so as to improve the image quality compared with IR algorithms (18-21). In our study, we extended the study to pediatric patients with TAK reconstructed with DLIR at the high setting (DLIR-H) to investigate whether DLIR-H could improve image quality under normal scan conditions in comparison with the traditional Filter back projection (FBP) and the state-of-the-art adaptive statistical iterative reconstruction (ASIR-V) algorithm.

\section{Methods}

\subsection{Patients}

The study was conducted in accordance with the Declaration of Helsinki (as revised in 2013). This was a retrospective study approved by the ethics committee of our hospital, and informed consents from patients were waived. The CTA was performed continuously from Nov 1, 2019 to Jun 30, 2020.

\subsection{CT scan and image reconstruction}

All examinations were performed on a 256 rows multi-detector CT scanner (Revolution CT, GE Healthcare, USA), using a tube voltage of $100 \mathrm{kVp}$, helical pitch value of $1.375: 1$ with $40 \mathrm{~mm}$ detector width and $0.5 \mathrm{~s}$ rotation speed. The automatic tube current modulation technique was used with tube current in the range of 50-500mA, noise index was 11 . All scans were performed when children were in a quiet state. For those children who could not cooperate, the $10 \%$ chloral hydrate at a dose of $0.4 \mathrm{ml} / \mathrm{kg}$ based on body weight was given orally and scans would not start until patients fell asleep. The scanning range included neck, chest, and abdomen, with the upper edge from the skull base and the lower edge to the anterior superior iliac spine.

For the contrast-enhanced CT protocol, a peripheral venous cannula was pre-placed in the superficial vein of dorsum of the hand. An iodinated contrast agent ( $320 \mathrm{mg} \mathrm{l} / \mathrm{ml}$ iodixanol; GE Healthcare, USA) was administered using a single-head power injector. The contrast medium dosage was calculated according to the body weight of each child: $1.6 \mathrm{ml} / \mathrm{kg}$ for children lower than $16.0 \mathrm{~kg}, 1.4 \mathrm{ml} / \mathrm{kg}$ for $16.1-35.0 \mathrm{~kg}$ and $1.2 \mathrm{ml} / \mathrm{kg}$ for higher than $35.0 \mathrm{~kg}$ (the maximum usage limit was $70 \mathrm{ml}$ ). Flow rate was adjusted according to a fixed injection time of $15 \mathrm{~s}$ and contrast enhanced scan started at $17 \mathrm{~s}$ after the start of contrast injection.

The raw data was reconstructed to $0.625 \mathrm{~mm}$ thin slice images using 3 reconstruction algorithms into 4 groups: FBP group, 50\%ASIR-V group, 100\%ASIR-V group, and DLIR-H group. All reconstructions used a standard kernel.

\subsection{Image quality evaluation}


All images were transferred to an advantage workstation (AW4.7, GE Healthcare, USA). Two observers with 15 and 8 years of experience in reviewing CTA images subjectively evaluated image quality using a 5-point scoring system. The reviewers were aware of the clinical task of diagnosing TA but were blinded to patient information and reconstruction algorithms. The readers evaluated all 4 differently reconstructed images of the same patient before moving to different patients, but the order in which the different reconstructions appear on the AW changed randomly. The observers could freely adjust the window width and position for observation and used multiplanar reconstructions (MPR) and threedimensional images for the evaluation. If the scores given by the two observers were not the same, a third senior doctor with 20 years of experience in reviewing children chest CT images would evaluate the images and gave the final score. Subjective scores included the overall image noise, visualization of large arteries (artery diameter greater than the vertebral artery or proper hepatic artery), visualization of small arteries (artery diameter less than or equal to the vertebral artery or proper hepatic artery) and diagnostic confidence. Scores with at least 3 points were accepted for diagnosis with 5 points being the best. The specific evaluation criteria are listed in Table 1.

Table 1

specific criteria for subjective evaluation

\begin{tabular}{|llllll|}
\hline & 1 point & 2 points & 3 points & $\begin{array}{l}\mathbf{4} \\
\text { points }\end{array}$ & 5 points \\
\hline Overall image noise & severe & high & moderate & little & rare \\
\hline $\begin{array}{l}\text { Vessel display ability } \\
\text { non- } \\
\text { detect } \\
\text { confiagnostic }\end{array}$ & none & little & moderate & high & definite \\
\hline
\end{tabular}

After the subjective evaluation, the two observers conducted objective measurement on the AW workstation together, selected the largest section of the liver portal, set a circular region of interest (ROI) on the descending aorta (Ao) on the liver portal section with a diameter half of that of Ao and on the back muscle $(\mathrm{Mu})$ at the same imaging level to measure their $\mathrm{CT}$ attenuation value and standard deviation (SD) value. The contrast-to-noise ratio (CNR) for the descending aorta was calculated using the following formula:

$\mathrm{CNR}=(\mathrm{CT}(\mathrm{Ao})-\mathrm{CT}(\mathrm{Mu})) /((\mathrm{SD}(\mathrm{Ao})+\mathrm{SD}(\mathrm{Mu})) / 2)$

\subsection{Statistical analysis}

All the data were expressed using the form of mean \pm standard deviation. The differences of the 4 image groups were analyzed, continuous variables following the normal distribution were analyzed by using the repeated measures analysis of variance with Bonferroni correction. The ordinal scales or variables that failed to follow normal distribution were analyzed by using Friedman's test. $\mathrm{P}<0.05$ was considered to have statistically significant difference. 


\section{Results}

A total of 32 patients (9 males and 23 females) with an average age of $9.14 \pm 4.51$ years (1-17 years) were included in the study. The volume CT dose index (CTDIvol) for the group was $4.53 \pm 2.62 \mathrm{mGy}$ and the dose-length-product (DLP) was $258.39 \pm 189.93 \mathrm{mGy} . \mathrm{cm}$.

The results of the subjective evaluation and objective measurement are listed in Table 2 and Table 3, respectively. For the subjective score, the overall image noise of the $50 \% A S I R-V, 100 \% A S I R-V$ and DLIR-H images could meet the diagnostic requirements, with the 100\%ASIR-V and DLIR-H images having the highest scores, and there was no statistical difference between them; for the visualization of large arteries, all images could met the diagnostic requirements (Fig. 1), and 100\%ASIR-V and DLIR-H images were the best, and there was no statistical difference between them; for the visualization of small arteries, the $100 \% A S I R-V$ over-smoothed images with sub-optimal resolution $(2.84 \pm 0.37)$ and only the $50 \% A S I R-V$ and DLIR-H could provide the satisfactory diagnostic confidence for all vessels, with DLIR-H being the best reconstruction algorithm (Fig. 2); The DLIR-H provided significantly better diagnostic confidence $(4.09 \pm 0.30)$ than that of $50 \% A S I R-V(3.03 \pm 0.18)$ and $100 \% A S I R-V(3.00 \pm 0.00)(p<0.001)$. For the objective measurement results: there was no statistical difference in the vascular CT number and muscle CT number among the reconstruction groups; for the vascular noise and muscle noise, the values with $100 \% A S I R-V(18.46 \pm 3.91 \mathrm{HU}$ and $11.22 \pm 2.40 \mathrm{HU})$ and DLIR-H $(15.58 \pm 3.65 \mathrm{HU}$ and $12.64 \pm 2.71 \mathrm{HU})$ had no statistically significant difference between them, and both significantly lower those $(24.96 \pm 4.69 \mathrm{HU}$ and $17.68 \pm 3.52 \mathrm{HU})$ of $50 \% A S I R-V$ images. For CNR, the values with $100 \% A S I R-V(22.54 \pm 3.34)$ and DLIR-H $(24.02 \pm 4.55)$ also had no statistically significant difference between them and were both significantly higher than that $(15.66 \pm 2.37)$ with $50 \% A S I R-V$. Compared with the FBP images, DLIR-H images reduced image noise by $50.32 \%$ in vessels and $49 \%$ in soft tissue, and the $50 \% A S I R-V$ reduced images noise by $20.41 \%$ in vessels and $28.68 \%$ in soft tissue; For CNR, DLIR-H increased the value by $101.34 \%$ and $50 \%$ ASIR-V by $88.94 \%$.

Table 2

Results of subjective scores

\begin{tabular}{|llllll|}
\hline & FBP & 50\%ASIR-V & 100\%ASIR-V & DLIR & $\begin{array}{l}\text { P } \\
\text { value }\end{array}$ \\
\hline Overall image noise & $2.16 \pm 0.37$ & $3.09 \pm 0.30$ & $4.41 \pm 0.50^{*}$ & $4.03 \pm 0.18^{*}$ & $<$ \\
& & & & & 0.001 \\
\hline $\begin{array}{l}\text { Display ability of large } \\
\text { arteries }\end{array}$ & $3.06 \pm 0.25$ & $4.03 \pm 0.31^{*}$ & $4.59 \pm 0.50 \#$ & $4.34 \pm$ & $<$ \\
\hline $\begin{array}{l}\text { Display ability of small } \\
\text { arteries }\end{array}$ & $2.94 \pm$ & $3.03 \pm$ & $2.84 \pm$ & $3.53 \pm 0.51$ & $<$ \\
Diagnostic confidence & $0.25^{\star} \#$ & $0.18^{\star}+$ & $0.37 \#+$ & & 0.001 \\
& $2.91 \pm$ & $3.03 \pm$ & $3.00 \pm$ & $4.09 \pm 0.30$ & $<$ \\
\hline *\#+: without in-group statistical difference & & $0.00 \#+$ & & 0.001 \\
\hline
\end{tabular}


Table 3

Results of objective scores

\begin{tabular}{|llllll|}
\hline & FBP & 50\%ASIR-V & 100\%ASIR-V & DLIR & P value \\
\hline Artery CT value* & $391.27 \pm 55.23$ & $391.92 \pm 54.48$ & $392.28 \pm 53.79$ & $393.61 \pm 54.19$ & 1.00 \\
\hline Artery SD value & $31.36 \pm 6.01$ & $24.96 \pm 4.69$ & $18.46 \pm 3.91^{*}$ & $15.58 \pm 3.65^{*}$ & $<0.001$ \\
\hline Muscle CT value* & $60.46 \pm 9.38$ & $60.42 \pm 8.74$ & $60.22 \pm 8.10$ & $60.54 \pm 7.69$ & 1.00 \\
\hline Muscle SD value & $24.79 \pm 5.16$ & $17.68 \pm 3.52$ & $11.22 \pm 2.40^{*}$ & $12.64 \pm 2.71^{*}$ & $<0.001$ \\
\hline Artery CNR & $11.93 \pm 2.12$ & $15.66 \pm 2.37$ & $22.54 \pm 3.34^{*}$ & $24.02 \pm 4.55^{*}$ & $<0.001$ \\
\hline CNR: contrast to noise ratio & & & & \\
\hline *: without in-group statistical difference & & & & \\
\hline
\end{tabular}

\section{Discussion}

In our study we investigated whether DLIR-H could improve image quality in CTA of pediatric patients with TAK under normal scan conditions, in comparison with the traditional FBP and the state-of-the-art $50 \% A S I R-V$ algorithm. From the objective evaluation point of view, our results showed that DLIR-H provided about $50 \%$ image noise reduction, while 50\%ASIR-V provided about $25 \%$ reduction compared with FBP. From the subjective evaluation point of view, our results showed that DLIR-H did not affect the visualization of small arteries and image texture while reducing image noise. The overall appearance of DLIR-H images was similar to that of FBP and 50\%ASIR-V images, without "blotchy" feeling, which was conducive to the display of detailed structures such as small arteries. In fact, since the overall image noise was significantly reduced, the ability of displaying small vessels was judged to be even better than that of FBP and 50\%ASIR-V (3.53 vs. 2.94 and 3.03). All the subjective image quality scores of DLIR-H were higher than 3.0, and its comprehensive score was better than other reconstruction algorithms. On the other hand, even though $100 \%$ ASIR-V also significantly reduced image noise, it also altered image texture making images appeared too "smooth" with some spatial resolution and structure detail loses (Fig. 2), which affected the subjective diagnostic confidence and the diameter measurement for small arteries.

CTA is an important evaluation method for TAK (18-19). Since TAK can involve the whole artery system, the scanning range is general much larger (including neck, chest and abdomen simultaneously) than that of conventional CTA. In addition, it is also necessary to observe whether the arterial branches with small arteries are involved, high spatial resolution and thin-layer images are desirable. All these clinical requirements put more pressure on the radiation dose in CTA for TAK patients (22), and efficient solutions are urgently needed to reduce the radiation dose while maintaining or improving image quality.

IR algorithms have been widely used to tackle the problem of higher image noise with thin-layer images and/or under reduced radiation conditions. However, IR algorithms with high weights tend to over smooth 
the high frequency noise and reduce the average frequency of the noise power spectrum (NPS) of the images. Although the image noise is greatly reduced, images may lose the normal image texture (Fig. 2), making images look "overly smooth", "plastic", or simply "unnatural" $(16,17,23)$. Our results indicated that although it did not affect the visualization of large arteries (Fig. 1), it was not conducive to display image details such as small arteries (Fig. 2) using 100\%ASIR-V algorithm. Therefore, in general, 50\% weight of IR is recommended to maintain the balance between noise and image resolution in clinical routine work. Only when the radiation dose is very low, $100 \%$ IR weighted is recommended as a mean to salvage the scans (24). This is the major limitation of low-dose CTA applicated in TAK. DLIR is a new generation of image reconstruction algorithm. The deep learning method is used to analyze the characteristics of image noise generation and distribution in different organizations. In theory, the targeted reduction of noise will have minimum impact on the image resolution. In the process of DLIR reconstruction, the integrity of the image information remains largely unchanged when the image noise is greatly reduced, and there is no artificial addition or loss of image resolution. Our results indicated that when DLIR algorithm was used in the assessment of TAK, the image details were maintained, while image noise was significantly reduced. Ever though our study was focused on image noise reduction and image quality improvement, since at the current dose level with $50 \% A S I R-V$, image quality was acceptable, the lower image noise and better image quality performance of DLIR-H could be traded with radiation dose reduction in the future.

There were still some limitations in this study. Firstly, the case number in the study was rather small, because TAK is not a common disease, so it was hard to collect large samples, and impossible to compare children of different ages in groups. In the future, the sample size will be increased to enrich the data information. Secondly, subjective image evaluation was performed using a non-validated scale developed by the authors. Third, our study focused on the image quality improvement over the state-ofthe-art ASIR-V algorithm using the same scan under the standard-dose condition. Although the image quality of some ASIR-V images was different from those of DLIR images, no children had interventional radiology results as the gold standard, so it was impossible to confirm the diagnostic accuracies of the different reconstruction algorithms. Further research is needed to demonstrate the improvement in diagnostic efficacy. In addition, further research is also needed to focus on dose reduction to investigate

whether DLIR could achieve the same image quality and diagnostic efficacy as the standard-dose ASIR-V images under lower-radiation dose or lower-contrast agent dose.

\section{Conclusion}

DLIR-H improves the CTA image quality and diagnostic confidence for TAK patients compared with the state-of-the-art 50\%ASIR-V, and best balances image noise and spatial resolution compared with $100 \%$ ASIR-V.

\section{Abbreviations}

ALARA: as low as reasonably achievable 
CNR: contrast-to-noise ratio

CTA: CT angiography

CTDIvol: CT dose index

DLIR: Deep learning image reconstruction

DLIR-H: DLIR with high setting

DLP: dose-length-product

FBP: Filtered Back-Projection

NBP: noise power spectrum

SD: standard deviation

TAK: Takayasu arteritis

\section{Declarations}

\section{Acknowledgments}

The authors would like to thank Dr. Z.L., a senior cardiovascular radiologist, for helping us to produce the final subjective evaluation results in case of disagreement between the two observers.

\section{Author Contribution}

H.L. ${ }^{5}$ and Y.P. designed the study, drafted the initial manuscript, and reviewed and revised the manuscript. J.S., H.L. 1, J.L., Z.Z., and M.L. coordinated and supervised data collection, and critically reviewed the manuscript for important content. All authors have read and approved the manuscript, and ensure that this is the case.

\section{Funding}

This research did not receive any specific grant from funding agencies in the public, commercial, or notfor-profit sectors.

\section{Availability of data and materials}

The data is available from the corresponding author on reasonable request.

Ethics approval and consent to participate 
The authors are accountable for all aspects of the work in ensuring that questions related to the accuracy or integrity of any part of the work are appropriately investigated and resolved. The study was conducted in accordance with the Declaration of Helsinki (as revised in 2013). This study was approved by the Ethical Committee of our Hospital. Informed consents were waived by Ethical Committee of our Hospital.

\section{Consent for publication}

Not Applicable.

\section{Competing interests}

Dr. J.L. reports that he is an employee of GE Healthcare, the manufacturer of the CT system used in this study. The other authors have no conflicts of interest to declare.

\section{References}

1. Tola H, Ozturk E, Yildiz O, et al. Assessment of Children with Vascular Ring. Pediatr Int 2017;59:134140.

2. Backer CL, Mongé MC, Popescu AR, et al. Vascular Rings. Semin Pediatr Surg 2016;25:165-175.

3. Tashtoush B, Memarpour R, Gonzalez J, et al. Pulmonary Sequestration: A 29 Patient Case Series and Review. J Clin Diagn Res 2015;9:AC05-8.

4. Trotman-Dickenson B. Congenital lung disease in the adult: guide to the evaluation and management. J Thorac Imaging 2015;30:46-59.

5. Kim HJ, Shin KE, Park JS, et al. Intralobar Pulmonary Sequestration with Cystic Degeneration Mimicking a Bronchogenic Cyst in an Elderly Patient: A Case Report and Literature Review. Medicine (Baltimore) 2020;99:e19347.

6. Zhang N, Zeng Q, Chen C, et al. Distribution, Diagnosis, and Treatment of Pulmonary Sequestration: Report of 208 Cases. J Pediatr Surg 2019;54:1286-1292.

7. Hermelijn CM, Elders BB, Ciet P, et al. A Clinical Guideline for Structured Assessment of CT-imaging in Congenital Lung Abnormalities. Paediatr Respir Rev 2020;20:S1526-0542(20)30019-1.

8. Duftner C, Dejaco C, Sepriano A, et al. Imaging in diagnosis, outcome prediction and monitoring of large vessel vasculitis: a systematic literature review and meta-analysis informing the EULAR recommendations. RMD Open 2018;4:e000612.

9. Bardi M, Diamantopoulos AP. EULAR recommendations for the use of imaging in large vessel vasculitis in clinical practice summary. Radiol Med 2019;124:965-972.

10. Direskeneli H, Aydin SZ, Merkel PA. Assessment of disease activity and progression in Takayasu's arteritis. Clin Exp Rheumatol 2011;29:S86-91.

11. Mukhtyar C, Guillevin L, Cid MC, et al. EULAR recommendations for the management of large vessel vasculitis. AnnRheum Dis 2019;8:318-323. 
12. The ALARA (as low as reasonably achievable) concept in pediatric CT intelligent dose reduction. Multidisciplinary conference organized by the Society of Pediatric Radiology. August 18-19, 2001. Pediatr Radiol 2002;32:217-313.

13. Schindera ST, Diedrichsen L, Müller HC, et al. Iterative Reconstruction Algorithm for Abdominal Multidetector CT at Different Tube Voltages: Assessment of Diagnostic Accuracy, Image Quality, and Radiation Dose in a Phantom Study. Radiology 2011;260:454-462.

14. Goenka AH, Herts BR, Dong F, et al. Image Noise, CNR, and Detectability of Low-Contrast, LowAttenuation Liver Lesions in a Phantom: Effects of Radiation Exposure, Phantom Size, Integrated Circuit Detector, and Iterative Reconstruction. Radiology 2016;280:475-482.

15. Yan C, Xu J, Liang C, et al. Radiation Dose Reduction by Using CT With Iterative Model Reconstruction in Patients With Pulmonary Invasive Fungal Infection. Radiology 2018;288:285-292.

16. Greffier J, Frandon J, Larbi A, et al. CT iterative reconstruction algorithms: a task-based image quality assessment. Eur Radiol 2020;30:487-500.

17. Mileto A, Guimaraes LS, McCollough CH, et al. State of the Art in Abdominal CT: The Limits of Iterative Reconstruction Algorithms. Radiology 2019;293:491-503.

18. Benz DC, Benetos G, Rampidis G, et al. Validation of Deep-Learning Image Reconstruction for Coronary Computed Tomography Angiography: Impact on Noise, Image Quality and Diagnostic Accuracy. J Cardiovasc Comput Tomogr 2020;13:S1934-5925(19)30464-2.

19. Akagi M, Nakamura Y, Higaki T, et al. Deep Learning Reconstruction Improves Image Quality of Abdominal Ultra-High-Resolution CT. Eur Radiol 2019;29:6163-6171.

20. Tatsugami F, Higaki T, Nakamura Y, et al. Deep Learning-Based Image Restoration Algorithm for Coronary CT Angiography. Eur Radiol 2019;29:5322-5329.

21. Greffier J, Hamard A, Pereira F, et al. Image quality and dose reduction opportunity of deep learning image reconstruction algorithm for CT: a phantom study. Eur Radiol 2020;30:3951-3959.

22. Masuda T, Funama Y, Nakaura T, et al. Radiation Dose Reduction with a low-tube voltage technique for pediatric chest computed tomographic angiography based on the contrast-to-noise ratio index. Can Assoc Radiol J 2018;69:390-396.

23. Geyer LL, Schoepf UJ, Meinel FG, et al. State of the art: iterative CT reconstruction techniques. Radiology 276:339-357.

24. Sun J, Yang L, Zhou Z, et al. Performance evaluation of two iterative reconstruction algorithms, MBIR and ASIR, in low radiation dose and low contrast dose abdominal CT in children. Radiol Med 2020;125:918-925.

\section{Figures}




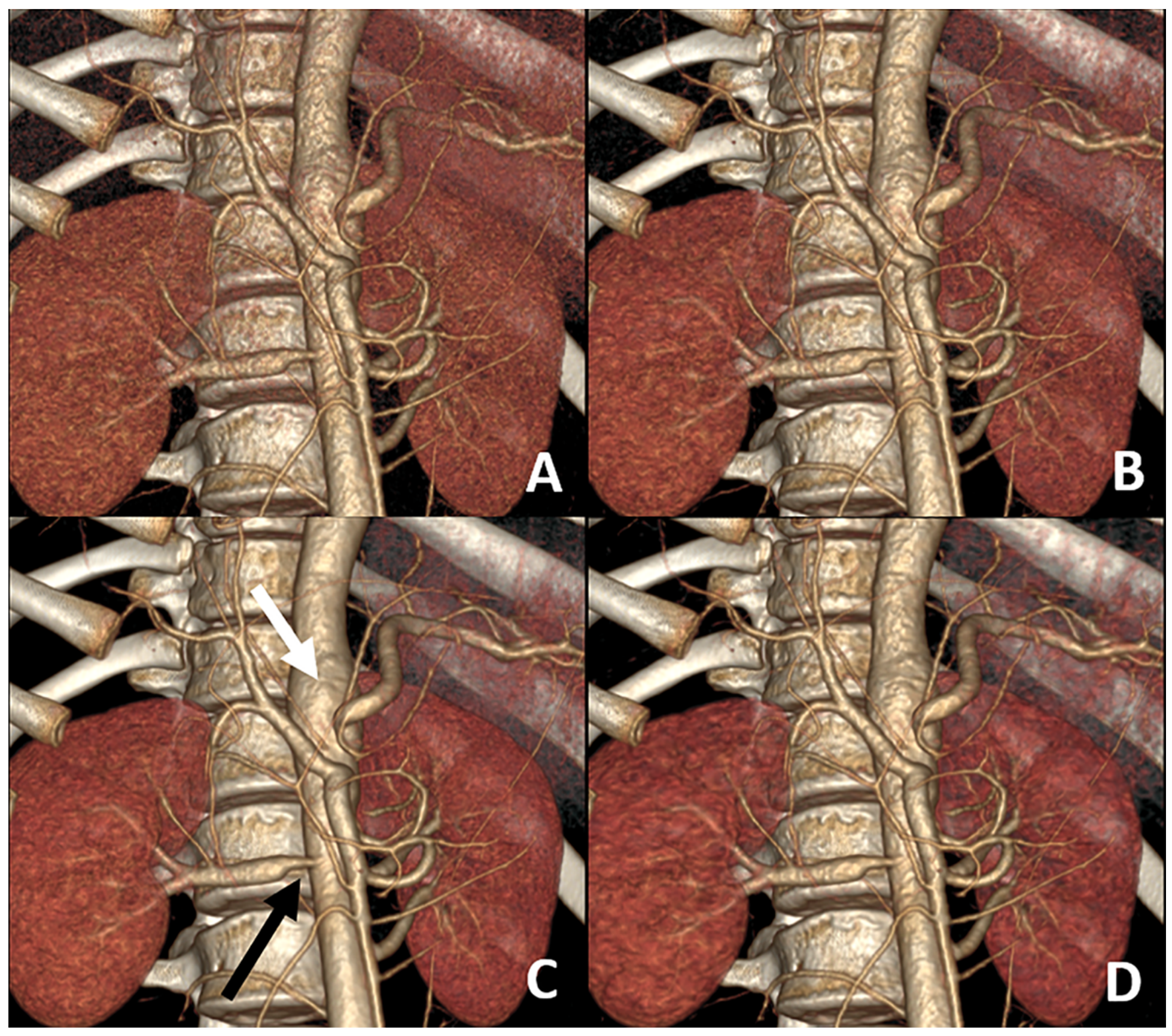

Figure 1

Images of a 14-years old girl suffered from Takayasu arteritis. The scan voltage was 100kV, 1A-1D was the $0.625 \mathrm{~mm}$ image with FBP, $50 \% A S I R-V, 100 \% A S I R-V$ and DLIR-H. respectively. The image noise of $1 \mathrm{C}$ and $1 \mathrm{D}$ were lower than that of $1 \mathrm{~A}$ and $1 \mathrm{~B}$ (* area). All images could show the slight dilation of big arteries (white arrow), and the stenosis of right renal artery (black arrow), 1C and 1D images had the least image noise. 


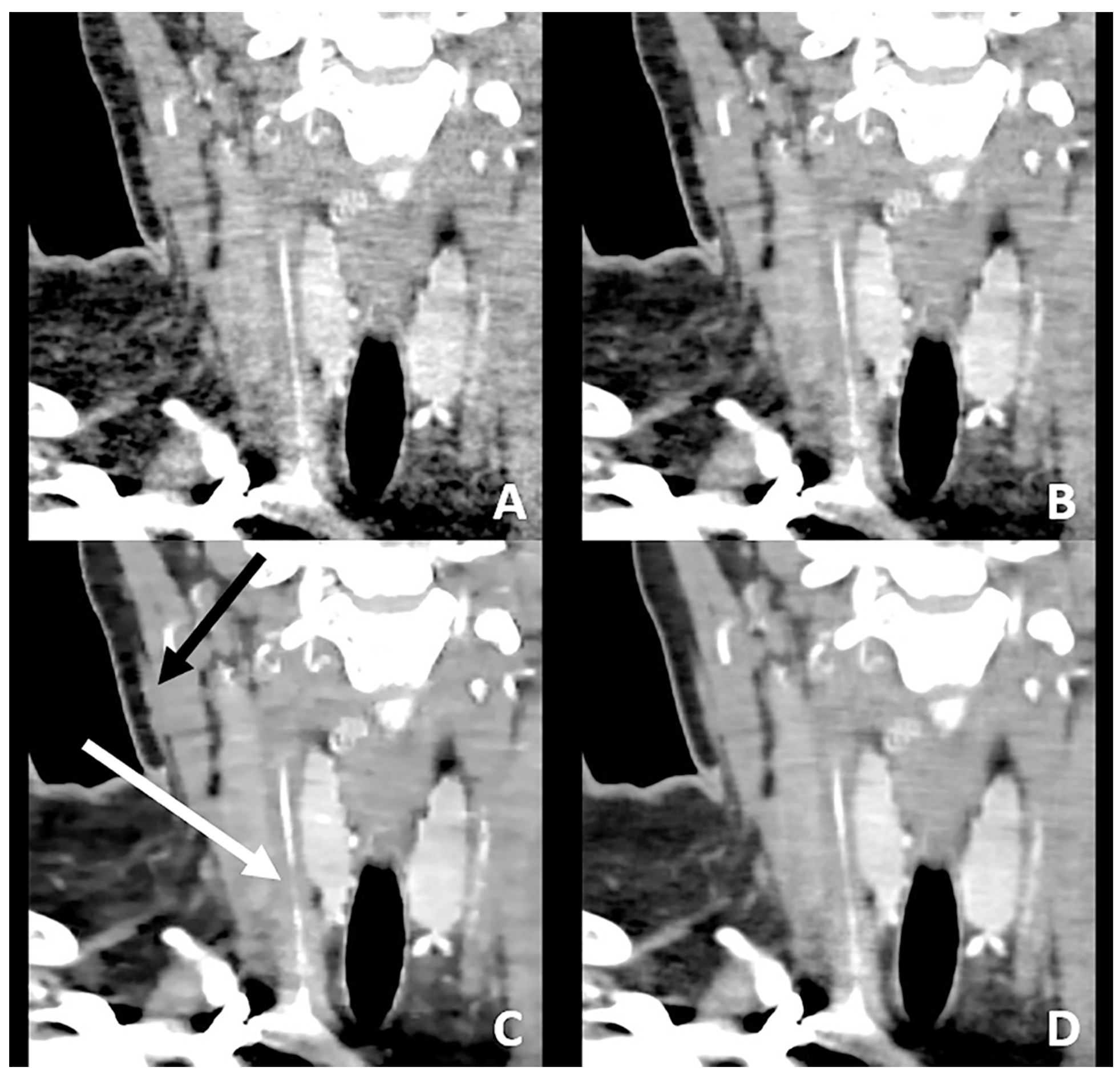

Figure 2

Images of a 14-years old girl suffered from Takayasu's arteritis. The scan voltage was 100kV, 2A-2D was the $0.625 \mathrm{~mm}$ image with FBP, $50 \% A S I R-V, 100 \% A S I R-V$ and DLIR-H, respectively. The $100 \% A S I R-V$ image (2C) had the least image noise but was also blotchy in the image texture (black arrow), the boundary of muscles was unclear compared to 2D. The margin of the small artery was blurred and not consistent (white arrow), which could induce the misdiagnosis as vascular occlusion. DLIR-H image (2D) had similar low image noise as $2 \mathrm{C}$ but without the pixilated appearance seen in $2 \mathrm{C}$ and provided the highest diagnostic confidence for the vessels. 